\title{
Critical dimension and negative specific heat in one-dimensional large- $N$ reduced models
}

\author{
Takeshi Morita $^{1,2, *}$ and Hiroki Yoshida ${ }^{1,2, \uparrow}$ \\ ${ }^{1}$ Department of Physics, Shizuoka University, 836 Ohya, Suruga-ku, Shizuoka 422-8529, Japan \\ ${ }^{2}$ Graduate School of Science and Technology, Shizuoka University, \\ 836 Ohya, Suruga-ku, Shizuoka 422-8529, Japan
}

(Received 20 January 2020; accepted 9 April 2020; published 11 May 2020)

\begin{abstract}
We investigate critical phenomena of the Yang-Mills (YM) type one-dimensional matrix model that is a large- $N$ reduction (or dimensional reduction) of the $D+1$ dimensional $U(N)$ pure YM theory (bosonic Banks-Fischler-Shenker-Susskind model). This model shows a large- $N$ phase transition at finite temperature, which is analogous to the confinement/deconfinement transition of the original YM theory. We study the matrix model at a three-loop calculation via the "principle of minimum sensitivity" and find that there is a critical dimension $D=35.5$ : At $D \leq 35$, the transition is of first order, while it is of second order at $D \geq 36$. Furthermore, we evaluate several observables in our method, and they nicely reproduce the existing Monte Carlo results. Through the gauge/gravity correspondence, the transition is expected to be related to a Gregory-Laflamme transition in gravity, and we argue that the existence of the critical dimension is qualitatively consistent with it. Besides, in the first order transition case, a stable phase having negative specific heat appears in the microcanonical ensemble, which is similar to Schwarzschild black holes. We study some properties of this phase.
\end{abstract}

DOI: $10.1103 /$ PhysRevD.101.106010

\section{INTRODUCTION}

Critical phenomena in physics sometimes show interesting dependences on the numbers of the spatial dimensions. One remarkable example is the Gregory-Laflamme (GL) transition in the $D+1$ dimensional gravity with a compact $S^{1}$ circle [1]. (See a review [2].) By changing the size of the $S^{1}$ from small to large, the stable configuration for a given energy changes from a uniform black string (UBS) to a localized black hole (LBH), and this transition is called the GL transition. A nonuniform black string (NUBS) may appear as an intermediate state in this transition. Surprisingly, the order of this phase transition does depend on $D$, and it is of first order at $D \leq 12$, while is of second order at $D \geq 13$ [3]. Hence, $D=12.5$ can be regarded as a critical dimension of this transition. Curiously, if we fix the temperature instead of the energy, the critical dimension changes to $D=11.5$ [4]. See Table I.

A similar critical dimension appears in the RayleighPlateau (RP) instabilities in liquid too. If we consider a

\footnotetext{
*morita.takeshi@shizuoka.ac.jp

yoshida.hiroki.16@shizuoka.ac.jp
}

Published by the American Physical Society under the terms of the Creative Commons Attribution 4.0 International license. Further distribution of this work must maintain attribution to the author(s) and the published article's title, journal citation, and DOI. Funded by SCOAP. space time $\mathbb{R}^{D-1,1} \times S^{1}$ and set a liquid winding the $S^{1}$ with the same configuration as the UBS. Suppose that the volume of the liquid is fixed and the radius of the $S^{1}$ is increased. (Thus, the liquid is stretched along the $S^{1}$.) Then, above a critical radius, this configuration becomes unstable due to the RP instability, and it tends to be nonuniform. The order of this transition depends on $D$ similar to the GL transition, and it turned out that the critical dimension is $D=11.5[5,6]$. The connection between the GL and RP instabilities was also argued in [5].

According to the gauge/gravity correspondence [7,8], the GL transition is expected to be qualitatively related to the confinement/deconfinement (CD) transition in the $D+1$ dimensional Yang-Mills (YM) type matrix quantum mechanics, whose action at finite temperature is given by $[9-15]$

$S=\int_{0}^{\beta} d t \operatorname{Tr}\left\{\sum_{I=1}^{D} \frac{1}{2}\left(D_{t} X^{I}\right)^{2}-\sum_{I, J=1}^{D} \frac{g^{2}}{4}\left[X^{I}, X^{J}\right]^{2}\right\}$.

This model is a large- $N$ reduction (or dimensional reduction) of the $D+1$ dimensional $\mathrm{U}(N)$ pure Yang-Mills (YM) theory to one dimension [16]. Here $X^{I}(I=1, \ldots, D)$ are the $N \times N$ Hermitian matrices that are the dimensional reductions of the spatial components of the original $D+1$ dimensional gauge fields. $D_{t}:=\partial_{t}-i\left[A_{t},\right]$ is the covariant derivative and $A_{t}$ is the gauge field. $g$ is the coupling 
TABLE I. The critical dimensions of various models. The systems show the first order phase transitions below the critical dimensions and they become of second order above them. The critical dimension of the YM type matrix model is one of the main results of this article.

\begin{tabular}{lc}
\hline \hline & Critical dimension \\
\hline GL (fixed mass) & 12.5 \\
GL (fixed temperature) & 11.5 \\
RP & 11.5 \\
YM type matrix model (three-loop) & 35.5 \\
\hline \hline
\end{tabular}

constant, and we take the 't Hooft limit $N \rightarrow \infty$ and $g \rightarrow 0$ with a fixed 't Hooft coupling $\lambda:=g^{2} N$. Note that this model appears as low energy effective theories of D-branes and membranes in string theories in various situations, and is important in its own right $[9,17-22]$.

This model shows a large- $N$ phase transition [23,24], which is an analog of the $\mathrm{CD}$ transition of the original YM theory $[9,20,25-35]$. The order parameter of this transition is the Polyakov loop operators,

$$
u_{n}:=\frac{1}{N} \operatorname{Tr} \exp \left(\text { in } \int_{0}^{\beta} d t A_{t}\right), \quad(n=1,2, \ldots) .
$$

If $\left\langle u_{n}\right\rangle=0,(\forall n)$, it indicates a confinement, and, $\left\langle u_{n}\right\rangle \neq 0,(\exists n)$ shows a deconfinement.

The relation between the CD transition and the GL transition can be intuitively understood as follows. The diagonal components of $X^{I}$ can be regarded as the positions of $N$ particles (or D-branes). If we take the static diagonal gauge $\left(A_{t}\right)_{i j}=\alpha_{i} \delta_{i j}(i, j=1, \ldots, N), \alpha_{i}$ also describe the positions of the particles. (Here the configuration space of the gauge field is regarded as a real space.) Particularly, the Polyakov loop (2) is invariant under the shift $\alpha_{i}=\alpha_{i}+2 \pi / \beta$, and this space is actually an $S^{1}$ with the period $2 \pi / \beta$. At large- $N$, these particles may behave as a static fluid in the $D+1$ dimension [36], and their distribution would be uniform, nonuniform or localized along the $S^{1}$ as schematically shown in Fig. 1. Now the connection to the GL transition in the gravity is clear. These configurations would correspond to a UBS, NUBS

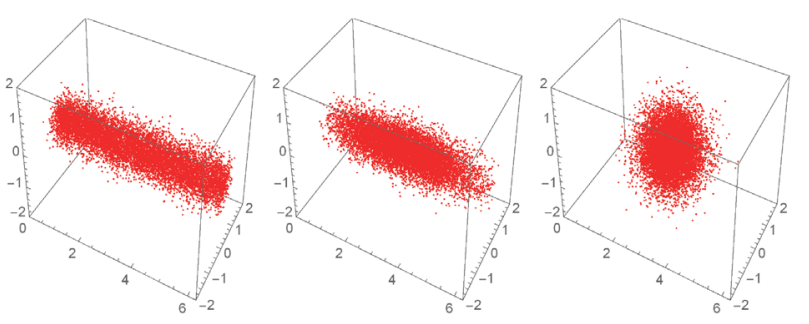

FIG. 1. Schematic plots of the "fluids" of the YM matrix model. Their distribution would be uniform, nonuniform or localized along the temporal circle direction. These are similar to the black string/black hole systems in gravity. and $\mathrm{LBH}$, respectively. Note that the temporal component $\alpha_{i}$ of the gauge theory corresponds to the spatial $S^{1}$ direction in the gravity [38]. As we have mentioned, the UBS is stable when the size of the $S^{1}$ is small. Correspondingly, the uniform distribution in Fig. 1 is stable at a small $2 \pi / \beta$, which means a low temperature. We can easily see that $\left\langle u_{n}\right\rangle=0$ in the uniform distribution, and this is consistent with the confinement at low temperatures. (The localized distribution is characterized by $u_{n} \neq 0$ for all $n$ and the nonuniform distribution is characterized by $u_{n} \neq 0$ for a finite number of $n$ 's. Thus, they are both deconfined.)

Since the critical dimensions appear in the GL and RP transitions, the existence of a critical dimension in the $\mathrm{CD}$ transition of the matrix model is expected. Indeed, several evidences for this conjecture have been found [31]. For small $D$, Monte Carlo (MC) simulations show that the order of the CD transition up to $D=25$ would be of first order $[31,34]$. On the other hand, at large- $D$, we can analyze the model analytically through the $1 / D$ expansion, and find the second order CD transition [30]. Hence, a critical dimension would exist in the matrix model too. In this article, we analyze the matrix model by using so-called "principle of minimum sensitivity" [39], and we will see that the critical dimension is $D=35.5$ at a three-loop calculation.

In addition, in the first order transition case, a phase having negative specific heat arises in the microcanonical ensemble $[24,40]$. Since some black holes such as Schwarzschild black holes and small black holes in AdS space-time [44] have negative specific heat too, the phases in the matrix model would be important to understand why these black holes have negative specific heat from the viewpoint of gauge theories [24]. Although, it is hard to explore such phases in MC calculations, we can easily access this phase in our method. We will derive several quantities in this phase near the critical temperature.

\section{ANALYSIS VIA THE PRINCIPLE OF MINIMUM SENSITIVITY}

To investigate the phase structure of the model (1), we employ the principle of minimum sensitivity [45]. Such a study was first done by Kabat and Lifschytz [25], but we use a different analysis in order to explore the details of the phase transition.

We deform the model (1) as

$$
\begin{aligned}
S & =S_{0}+\kappa S_{\mathrm{int}}, \\
S_{0} & =\int_{0}^{\beta} d t \operatorname{Tr}\left\{\sum_{I=1}^{D} \frac{1}{2}\left(D_{t} X^{I}\right)^{2}+\frac{M^{2}}{2}\left(X^{I}\right)^{2}\right\}, \\
S_{\text {int }} & =\int_{0}^{\beta} d t \operatorname{Tr}\left\{\sum_{I=1}^{D}-\frac{M^{2}}{2}\left(X^{I}\right)^{2}-\sum_{I, J=1}^{D} \frac{g^{2}}{4}\left[X^{I}, X^{J}\right]^{2}\right\} .
\end{aligned}
$$


Here we have introduced the deformation parameter $\kappa$ and $M$. If we take $\kappa=1$, the $M$ dependent terms are canceled, and this model goes back to the original model (1).

We integrate out $X^{I}$ through the perturbative calculations with respect to $\kappa$, and derive the effective action of the Polyakov loop $\left\{u_{n}\right\}$. The relevant terms at low temperatures, where all $u_{n}$ are small $[20,24]$, are given by

$$
\begin{aligned}
S_{\text {eff }}\left(\left\{u_{n}\right\}, M\right)= & N^{2}\left(\beta f_{0}+f_{1}\left|u_{1}\right|^{2}+f_{2}\left|u_{1}\right|^{4}\right. \\
& \left.+f_{3}\left|u_{2}\right|^{2}+f_{4}\left(u_{2} u_{-1}^{2}+u_{-2} u_{1}^{2}\right)+\cdots\right) .
\end{aligned}
$$

Here $f_{0}$ is a function of $M$ while $f_{i}(i=1,2,3,4)$ are functions of $M$ and $T:=1 / \beta$ [51]. The derivations and explicit expressions of $f_{0}$ and $f_{i}$ at three-loop order are shown in (A14)-(A18) in Appendix A. (If we are interested in the two-loop results [53], we simply remove the terms proportional to $\kappa^{2}$ in these equations.)

At this stage, we take $\kappa=1$. Although the initial model (3) at $\kappa=1$ is independent of the deformation parameter $M$, the obtained effective action does depend on $M$. Here, we fix $M$ so that the $M$ dependence of the effective action becomes a minimum. This prescription is so-called the principle of minimum sensitivity [39]. Although the validity of such a prescription is generally not ensured, it works very well for many examples. We will compare our results with the existing studies in order to test our analysis.

\section{A. Low temperature and confinement}

To explore the phase structure, we start from considering the low temperature regime. At low temperatures, we observe $f_{1}, f_{2}, f_{3}>0$ from (A15)-(A17). Then, the stable configuration in the effective action (4) is given by $u_{1}=u_{2}=0$, and it is in the confinement phase. Thus, we can approximate $S_{\text {eff }}=N^{2} \beta f_{0}$, and $M$ at low temperatures is fixed so that the $M$ dependence of $f_{0}$ is minimized, hence,

$$
\left|\partial_{M} f_{0}\left(M=M_{0}\right)\right|=\min \left|\partial_{M} f_{0}(M)\right|,
$$

where $M_{0}$ denotes the value of $M$ that minimizes $\left|\partial_{M} f_{0}\right|$. In the two-loop effective action, $f_{0}$ has a single extremum $\partial_{M} f_{0}=0$ via (A14), and it gives $M_{0}$ as

$$
M_{0}=\lambda^{1 / 3}(D-1)^{1 / 3}, \quad \text { (two-loop). }
$$

In the three-loop effective action, $f_{0}$ does not have any extremum. However, it has an inflection point $\partial_{M}^{2} f_{0}=0$, which minimizes (5), and we obtain

$$
M_{0}=\frac{15^{1 / 3} \lambda^{1 / 3}}{2}(D-3 / 4)^{1 / 3}, \quad \text { (three-loop). }
$$

In order to test whether these results are reliable, we evaluate the free energy $F:=S_{\text {eff }} / \beta=N^{2} f_{0}\left(M_{0}\right)$ and

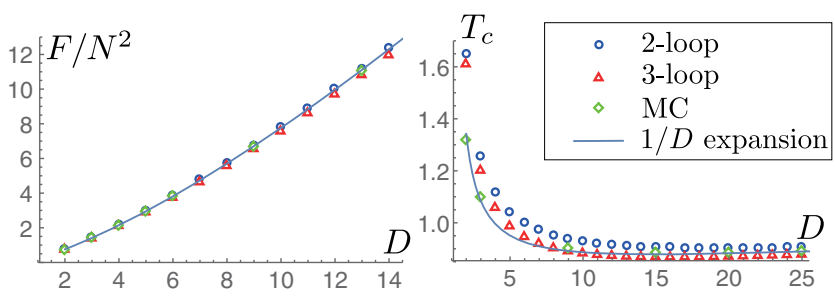

FIG. 2. Free energy $F / N^{2}$ in the confinement phase (the left panel) and critical temperature $T_{c}$ (the right panel). We have used the unit $\lambda=1$. The MC results are from [31,34]. The $1 / D$ expansion results are from (4.27) and (4.30) in [30]. In the MC results, we plot the transition temperature $T_{0}$ defined in Fig. 3, which should be slightly below $T_{c}$. We see good agreement in both of the plots.

compare them with the $\mathrm{MC}$ results at low temperatures [54]. By using (A14), we obtain $F$ in the confinement phase as

$$
F / N^{2}= \begin{cases}\frac{3}{8} D \lambda^{1 / 3}(D-1)^{1 / 3}, & \text { (two-loop) } \\ D \lambda^{1 / 3} \frac{(1412 D-1187)}{160(30(4 D-3))^{2 / 3}}, & \text { (three-loop). }\end{cases}
$$

These results are shown in Fig. 2 and Table II, and both the two- and three-loop analyses show good agreement [55].

Furthermore, in Appendix D, we also compare our results (8) at large- $D$ with the $1 / D$ expansion [30], which would provide reliable results there, and again find good agreement. Thus, we expect that our analysis via the principle of minimum sensitivity appropriately works in our model (1).

\section{B. Confinement/deconfinement transition}

As temperature increases, $f_{1}\left(M_{0}, T\right)$ becomes negative, and $u_{1}$ and $u_{2}$ may obtain nonzero vevs, indicating a deconfinement. This is the $\mathrm{CD}$ transition in our model. Near the critical temperature, $u_{1}$ and $u_{2}$ would be small and we can perturbatively treat them in the effective action (4). Correspondingly, $M$ can be expanded as

TABLE II. Free energy $F / N^{2}$ in the confinement phase. We have used the unit $\lambda=1$. The two-loop and three-loop results are from (8). The $1 / D$ expansion results are from (D1). The MC results are from the unpublished data in [31].

\begin{tabular}{lcccc}
\hline \hline$D$ & Two-loop & Three-loop & $1 / D$ expansion & MC $(T=0.50)$ \\
\hline 2 & 0.75 & 0.72 & 0.76 & $0.70(N=60)$ \\
3 & 1.42 & 1.37 & 1.41 & $1.42(N=32)$ \\
4 & 2.16 & 2.09 & 2.15 & $2.11(N=32)$ \\
5 & 2.98 & 2.88 & 2.95 & $2.93(N=24)$ \\
6 & 3.85 & 3.71 & 3.82 & $3.81(N=32)$ \\
9 & 6.75 & 6.52 & 6.71 & $6.66(N=32)$ \\
13 & 11.2 & 10.8 & 11.1 & $11.0(N=32)$ \\
\hline \hline
\end{tabular}




$$
\begin{aligned}
M= & M_{0}+M_{1}\left|u_{1}\right|^{2}+M_{2}\left|u_{1}\right|^{4} \\
& +M_{3}\left|u_{2}\right|^{2}+M_{4}\left(u_{2} u_{-1}^{2}+u_{-2} u_{1}^{2}\right)+\cdots
\end{aligned}
$$

Here, in the two-loop theory, $M_{0}$ is given by (6) and $M_{i}$ $(i=1, \ldots, 4)$ are fixed through the condition $\partial_{M} S_{\text {eff }}=0$ in (4). In the three-loop theory, $M_{0}$ is given by (7) and the condition $\partial_{M}^{2} S_{\text {eff }}=0$ determines $M_{i}$. (See the details in Appendix B.)

Then, by substituting (9) into the effective action (4) and using the small $\left\{u_{n}\right\}$ expansion, we obtain

$$
\begin{aligned}
S_{\mathrm{eff}}\left(\left\{u_{n}\right\}\right)= & N^{2}\left(\beta f_{0}+\bar{f}_{1}\left|u_{1}\right|^{2}+\bar{f}_{2}\left|u_{1}\right|^{4}\right. \\
& \left.+\bar{f}_{3}\left|u_{2}\right|^{2}+\bar{f}_{4}\left(u_{2} u_{-1}^{2}+u_{-2} u_{1}^{2}\right)+\cdots\right) .
\end{aligned}
$$

Here

$$
\begin{aligned}
& \bar{f}_{i}=f_{i}+\beta\left(\partial_{M} f_{0}\right) M_{i}, \quad(i=1,3,4), \\
& \bar{f}_{2}=f_{2}+\beta\left(\partial_{M} f_{0}\right) M_{2}+\frac{1}{2} \beta\left(\partial_{M}^{2} f_{0}\right) M_{1}^{2}+\left(\partial_{M} f_{1}\right) M_{1},
\end{aligned}
$$

where $f_{i}$ are evaluated at $M=M_{0}$. Finally, by integrating out $u_{2}$, we reach a Landau-Ginzburg type effective action for $u_{1}$,

$$
\begin{aligned}
S_{\text {eff }}\left(u_{1}\right) & =N^{2}\left(\beta f_{0}+a(T)\left|u_{1}\right|^{2}+b(T)\left|u_{1}\right|^{4}+\cdots\right), \\
a(T) & :=\bar{f}_{1}, \quad b(T):=\bar{f}_{2}-\frac{\bar{f}_{4}^{2}}{\bar{f}_{3}} .
\end{aligned}
$$

Although the explicit formulas for $a(T)$ and $b(T)$ are complicated and we omit to show them, it is straightforward to obtain them from (A14)-(A18) by using Mathematica.

Now we can easily see the phase structure $[20,24,26]$. If $a>0, u_{1}=0$ is (meta)stable and the system may be confined. If $a<0, u_{1}=0$ is unstable and $u_{1}$ has to develop a nonzero vev, and it is deconfinement. Thus, we can derive the critical temperature $T_{c}$ by solving $a\left(T_{c}\right)=0$. Numerical solutions of this equation are shown in Fig. 2 and Table III.

In order to determine the order of the transition, we expand $a(T)=-c\left(T-T_{c}\right)+\cdots(c:=\partial a / \partial T>0)$ near $T=T_{c}$, and obtain the classical solution of $u_{1}$ in (12) as

$$
u_{1}=\sqrt{-\frac{a}{2 b}} \simeq \sqrt{\frac{c\left(T-T_{c}\right)}{2 b}} .
$$

Therefore, if $b\left(T_{c}\right)$ is positive, it indicates a nontrivial solution in $T \geq T_{c}$, which implies a continuous second order phase transition. If $b\left(T_{c}\right)$ is negative, an unstable solution exists in $T \leq T_{c}$, and a first order phase transition
TABLE III. Critical temperature $T_{c}$. We have used the unit $\lambda=1$. The $1 / D$ expansion results are from (D3). The $\mathrm{MC}$ results are from [31] $(D \leq 20)$ and [34] $(D=25)$. In the MC results, we show the transition temperature $T_{0}$, which should be slightly below $T_{c}$.

\begin{tabular}{lcccl}
\hline \hline$D$ & Two-loop & Three-loop & $1 / D$ expansion & MC \\
\hline 2 & 1.65 & 1.61 & 1.34 & 1.32 \\
3 & 1.26 & 1.20 & 1.08 & 1.10 \\
9 & 0.938 & 0.889 & 0.892 & 0.901 \\
15 & 0.906 & 0.867 & 0.879 & 0.884 \\
20 & 0.903 & 0.869 & 0.882 & 0.884 \\
25 & 0.906 & 0.877 & 0.889 & 0.89 \\
\hline \hline
\end{tabular}

occurs at a temperature, which is slightly below $T_{c}$. We define this transition temperature as $T_{0}$. See Fig. 3.

\section{Critical dimension}

We plot $b\left(T_{c}\right)$ with respect to $D$ in Fig. 4. At two-loop order, $b$ is always negative and it predicts the first order phase transition. At three-loop order, $b$ becomes positive at $D=36$, and the transition changes to second order. Thus, the critical dimension of the model (1) is $D=35.5$ at three-loop.

\section{Phase with negative specific heat}

In the first order transition case, the unstable branch with $u_{1} \neq 0$ exists between $T_{1}$ and $T_{c}$ as shown in Fig. 3. Remarkably, it becomes stable in the microcanonical

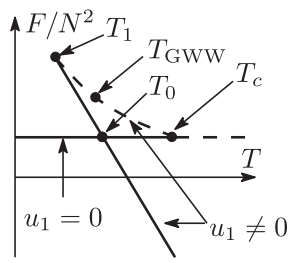

First order phase transition

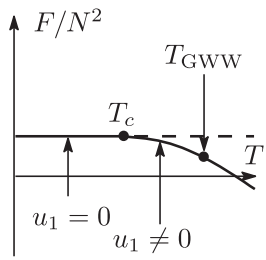

Second order phase transition
FIG. 3. Schematic plots of free energy vs temperature. The rigid lines depict stable and metastable phases, while the dashed lines depict unstable phases. The $u_{1}=0$ phase lines are horizontal and do not depend on $T$ due to the large- $N$ volume independence. In the first order phase transition case (the left panel), the unstable phase with $u_{1} \neq 0$ merges to the $u_{1}=0$ branch at $T=T_{c}$. Thus, the $u_{1} \neq 0$ solution (13) near $T_{c}$ exists in $T \leq T_{c}$. The phase transition occurs not at $T_{c}$ but at $T_{0}$ shown in the figure. In the second order phase transition case (the right panel), the stable $u_{1} \neq 0$ solution (13) appears in $T \geq T_{c}$. Therefore, through (13), the signature of $b$ at $T=T_{c}$ determines the order of the phase transition. Note that there is another transition point $T_{\mathrm{GWw}}$, at which a third order transition between the nonuniform distribution and the localized one in Fig. 1 occurs $[9,20,26,30]$. This transition is so-called the Gross-Witten-Wadia (GWW) transition [56,57], and is important in the context of the resolution of the naked singularities in the gravity $[26,58]$. 


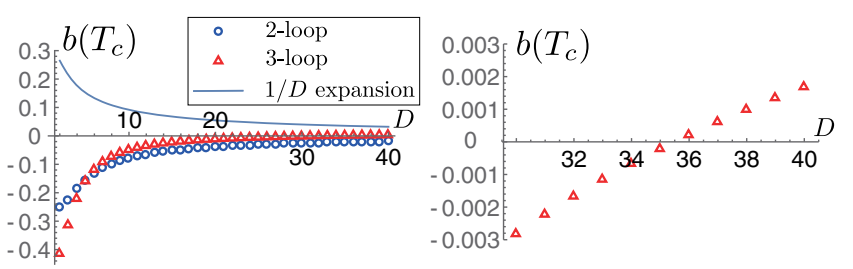

FIG. 4. The value of $b$ at the critical temperature. The negative and positive $b$ indicate the first and second order phase transitions, respectively. At three-loop, $b$ becomes positive at $D=36$, and hence the critical dimension is 35.5 . (See the right panel.) The $1 / D$ result [30] is from (D4). The MC simulations show first order transitions at least up to $D=25$ [31,34], and they are consistent with our result.
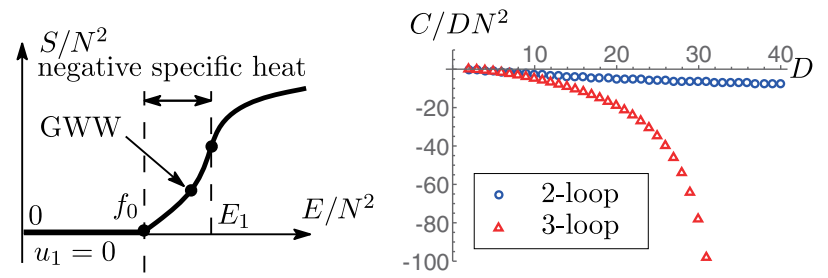

FIG. 5. (Left panel) A schematic plot of entropy vs energy in the microcanonical ensemble corresponding to the first order transition case in Fig. 3. The phase having negative specific heat characterized by $\partial^{2} S / \partial E^{2}>0$ appears between $E=N^{2} f_{0}$ and $E_{1}$, where $E_{1}$ is energy at $T=T_{1}$ in Fig. 3. The location of the GWW point would depend on $D$ and it may appear even above $E_{1}$. (Right panel) Negative specific heat $C$ at $T_{c}$ in the $u_{1} \neq 0$ phase. In the three-loop case, it diverges at the critical dimension, and the negative specific heat phase disappears in $D \geq 36$.

TABLE IV. Specific heat $C / D N^{2}$ of the $u_{1} \neq 0$ phase at $T=T_{c}$. It is positive in the second order phase transition case, while it is negative in the first order transition case. Also, it tends to diverge at the critical dimension $D=35.5$ in the three-loop case. The $1 / D$ expansion result is from (D5). The negative specific heat at $D=2$ in the $1 / D$ expansion is an error due to the failure of the expansion. At $D=2$, the subleading term in $b$ (D4) becomes larger than the leading term and it makes $1 / b$ negative after the expansion although $b$ is positive. Note that no one has succeeded in the computation of the specific heat at $T=T_{c}$ via MC.

\begin{tabular}{lccc}
\hline \hline$D$ & Two-loop & Three-loop & $1 / D$ expansion \\
\hline 2 & -0.4 & -0.25 & -0.45 \\
3 & -0.7 & -0.52 & 0.13 \\
9 & -2.6 & -4.2 & 2.4 \\
25 & -6.1 & -35.1 & 4.4 \\
35 & -7.4 & -930 & 5.0 \\
36 & -7.5 & 1069 & 5.1 \\
40 & -7.9 & 122 & 5.2 \\
100 & -11.7 & 16.2 & 6.7 \\
500 & -17.9 & 6.42 & 9.3 \\
\hline \hline
\end{tabular}

ensemble [24], and has negative specific heat akin to a Schwarzschild black hole. See the schematic plot in Fig. 5, where $\partial^{2} S / \partial E^{2}>0$ indicates the negative specific heat. (Here $S$ and $E$ are entropy and energy in the microcanonical ensemble.) We can read off the specific heat $C:=\partial_{T} E$ near $T=T_{c}\left(T \leq T_{c}\right)$ in this phase via (12) and (13) through the ordinary thermodynamical relations,

$$
\begin{aligned}
& F=N^{2} f_{0}-\frac{N^{2} c^{2} T_{c}}{4 b}\left(T-T_{c}\right)^{2}+\cdots, \\
& S=\frac{E-N^{2} f_{0}}{T_{c}}-\frac{b}{c^{2} T_{0}^{4}}\left(E-N^{2} f_{0}\right)^{2}+\cdots, \\
& C=\partial_{T} E=\frac{N^{2} T_{c}^{2} c^{2}}{2 b}+\cdots .
\end{aligned}
$$

Here $C$ is negative because $b<0$. We plot $C$ in Fig. 5 (right). See also Table IV. At three-loop, as $D$ approaches to the critical dimension $D=35.5, C$ diverges, since $b \rightarrow 0$.

\section{DISCUSSIONS}

We have shown that the critical dimension of the matrix model (1) is $D=35.5$ at three-loop. The existence of a critical dimension has been predicted through the MC [31] and the $1 / D$ expansion [30], and our result is consistent with them. Besides, the strong similarity between the GL, $\mathrm{RP}$ and the CD in the matrix model (1) are sharpened. This similarity may arise because the matrix model may describe a kind of fluid as depicted in Fig. 1. (The obtained critical dimension is different from the gravity, but it would not be a problem because we cannot expect any quantitative agreement in this correspondence [9,12].)

However, our analysis relies on the perturbative calculation and the principle of the minimum sensitivity, and $D=35.5$ is not conclusive. We need the higher order loop calculations to ensure it. At large- $D$, these corrections may make our results closer to those of the $1 / D$ expansion [30]. (See Appendix D for the results at large- $D$ in our analysis.)

Also, there are several varieties of the principle of the minimum sensitivity [46], and we need to check whether our results depend on these schemes.

Another remaining problem is understanding the properties of the first order phase transition at $T_{0}$ in $D \leq 35$. Above $T_{0}$, the stable configuration would be a nonuniform distribution or a localized one depending on $D$ [59]. If the stable configuration is a nonuniform distribution, another phase transition to a localized distribution must occur at a higher temperature. Indeed, these transitions have been found in the GL and RP transitions [2,6,60,61]. Besides, they would be important for a deeper understanding of the negative specific heat phase in the microcanonical ensemble.

In order to investigate them, we need to evaluate the effective action at finite $\left\{u_{n}\right\}$, and thus we cannot use the expansion (9). Besides, we need to calculate higher order 
couplings of the Polyakov loops such as $\left|u_{1}\right|^{6}$ in the effective action (4). We leave this problem for future work.

\section{ACKNOWLEDGMENTS}

We thank Y. Asano, T. Azuma, K. Hashimoto, G. Mandal, Y. Matsuo, K. Sugiyama and H. Suzuki for valuable discussions and comments. The work of T. M. is supported in part by Grant-in-Aid for Young Scientists B (No. 15K17643) from JSPS.

\section{APPENDIX A: THE EFFECTIVE ACTION (4) AT THREE-LOOP}

In this Appendix, we derive the effective action (4) at three-loop order. Starting from the deformed action (3), we compute the effective action of the Polyakov loop $\left\{u_{n}\right\}$ by integrating out $X^{I}$ through the standard perturbative calculation with respect to $\kappa$. It will lead to the expansion,

$$
S_{\text {eff }}\left(\left\{u_{n}\right\}, M\right)=\sum_{m=1}^{3} \kappa^{m-1} S_{m-\text { loop }}
$$

The analysis mainly follows that of the massive BanksFischler-Shenker-Susskind model [20]. In order to compute this expansion, we use the propagator of $X^{I}$ in the static diagonal gauge $\left(A_{t}\right)_{i j}=\alpha_{i} \delta_{i j}[30]$,

$$
\begin{aligned}
& \left\langle X_{i j}^{I}(t) X_{k l}^{J}(0)\right\rangle \\
& =\delta_{i l} \delta_{j k} \delta^{I J} \frac{1}{2 M} e^{i\left(\alpha_{i}-\alpha_{j}\right)\|t\|} \\
& \quad \times\left[e^{-M\|t\|} \sum_{n=0}^{\infty} x^{n} u_{n}^{i} u_{-n}^{j}+e^{M\|t\|} \sum_{n=1}^{\infty} x^{n} u_{-n}^{i} u_{n}^{j}\right] .
\end{aligned}
$$

Here $x:=e^{-\beta M}$ and $\|t\|$ denotes $\|t+n \beta\|=t$ for $0 \leq t<\beta$. $u_{n}^{i}:=e^{i \beta n \alpha_{i}}$, which satisfies $\sum_{i=1}^{N} u_{n}^{i}=N u_{n}$, where $u_{n}$ is the $n$th Polyakov loop defined in (2).

Through the one-loop integral, we obtain

$$
S_{1-\text { loop }} / N^{2}=\frac{D \beta M}{2}+\sum_{n=1}^{\infty} \frac{1-D x^{n}}{n}\left|u_{n}\right|^{2}
$$

At two-loop, we obtain
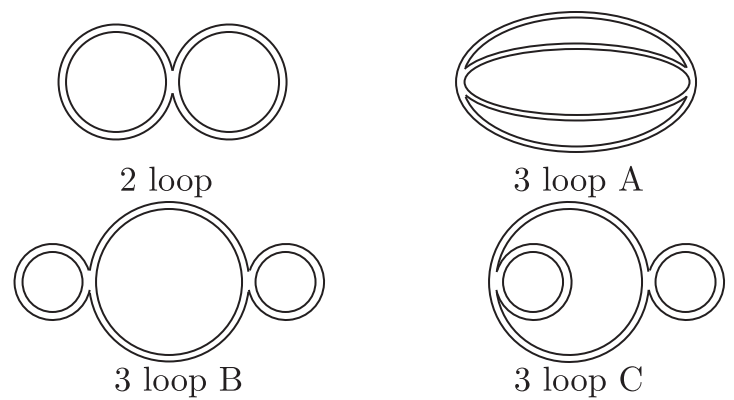

FIG. 6. Planar diagrams to compute the effective action. At three-loop order, the diagrams A, B and C correspond to $S_{3-1 o o p}^{A}$, $S_{3-\text { loop }}^{B}$ and $S_{3 \text {-loop }}^{C}$ in (A7), respectively.

$S_{2 \text {-loop }}=\left\langle\int_{0}^{\beta} d t \operatorname{Tr}\left(-\frac{g^{2}}{4}\left[X^{I}, X^{J}\right]^{2}-\frac{M^{2}}{2}\left(X^{I}\right)^{2}\right)\right\rangle$,

where

$$
\begin{aligned}
\left\langle\int_{0}^{\beta} d t \operatorname{Tr}\left(-\frac{g^{2}}{4}\left[X^{I}, X^{J}\right]^{2}\right)\right\rangle \\
=\frac{\beta N^{2} \lambda}{8 M^{2}} D(D-1)+\frac{\beta N^{2} \lambda}{4 M^{2}} D(D-1) \sum_{n=1}^{\infty}\left(x^{2 n}+2 x^{n}\right)\left|u_{n}\right|^{2} \\
\quad+\frac{\beta N^{2} \lambda}{8 M^{2}} D(D-1)\left(x^{2}+2 x^{3}\right)\left(u_{1}^{2} u_{-2}+u_{-1}^{2} u_{2}\right)+\cdots,
\end{aligned}
$$

$$
\begin{aligned}
& \left\langle\int_{0}^{\beta} d t \operatorname{Tr}\left(-\frac{M^{2}}{2}\left(X^{I}\right)^{2}\right)\right\rangle \\
& \quad=-M^{2} \frac{\partial}{\partial\left(M^{2}\right)} S_{1 \text {-loop }} \\
& \quad=-\frac{D N^{2} \beta M}{4}-\frac{D N^{2} \beta M}{2} \sum_{n=1}^{\infty} x^{n}\left|u_{n}\right|^{2} .
\end{aligned}
$$

Here (A5) has been computed via the planar diagram depicted in Fig. 6, and ... denotes the irrelevant terms at low temperatures. On the other hand, (A6) can be generated from the one-loop result (A3).

In order to compute the three-loop corrections, we need to evaluate

$$
-\frac{1}{2}\left\langle\int_{0}^{\beta} d t \operatorname{Tr}\left(-\frac{g^{2}}{4}\left[X^{I}, X^{J}\right]^{2}\right) \int_{0}^{\beta} d t^{\prime} \operatorname{Tr}\left(-\frac{g^{2}}{4}\left[X^{I}, X^{J}\right]^{2}\right)\right\rangle_{\text {connected }}=S_{3-\text { loop }}^{A}+S_{3 \text {-loop }}^{B}+S_{\text {3-loop }}^{C} .
$$

Here the last three terms are from the three diagrams depicted in Fig. 6, and we obtain 


$$
\begin{aligned}
& S_{3-\text { loop }}^{A} / N^{2}=-\beta \frac{3 \lambda^{2}}{128 M^{5}} D(D-1)-\frac{3 \beta \lambda^{2}}{32 M^{5}} D(D-1)\left(2 \beta M x^{2}-x^{3}+x^{2}+3 x\right)\left|u_{1}\right|^{2} \\
& -\frac{3 \beta \lambda^{2}}{64 M^{5}} D(D-1)\left(2 \beta M x^{2}-2 x^{4}+5 x^{2}\right)\left|u_{1}\right|^{4}-\frac{3 \beta \lambda^{2}}{32 M^{5}} D(D-1)\left(4 \beta M x^{4}-x^{6}+x^{4}+3 x^{2}\right)\left|u_{2}\right|^{2} \\
& -\frac{3 \beta \lambda^{2}}{32 M^{5}} D(D-1)\left(4 \beta M x^{3}-x^{5}-x^{4}+3 x^{3}+2 x^{2}\right)\left(u_{2} u_{-1}^{2}+u_{-2} u_{1}^{2}\right)+\cdots, \\
& S_{3-\text { loop }}^{B} / N^{2}=-\beta \frac{\lambda^{2}}{32 M^{5}} D(D-1)^{2}-\frac{\beta \lambda^{2}}{16 M^{5}} D(D-1)^{2}\left(\beta M\left(2 x^{2}+x\right)+3 x^{2}+3 x\right)\left|u_{1}\right|^{2} \\
& -\frac{3 \beta \lambda^{2}}{16 M^{5}} D(D-1)^{2}\left(\beta M x^{3}+x^{3}\right)\left|u_{1}\right|^{4}-\frac{\beta \lambda^{2}}{16 M^{5}} D(D-1)^{2}\left(2 \beta M\left(2 x^{4}+x^{2}\right)+3 x^{4}+3 x^{2}\right)\left|u_{2}\right|^{2} \\
& -\frac{\beta \lambda^{2}}{32 M^{5}} D(D-1)^{2}\left(2 \beta M\left(2 x^{4}+3 x^{3}+x^{2}\right)+3 x^{4}+6 x^{3}+3 x^{2}\right)\left(u_{2} u_{-1}^{2}+u_{-2} u_{1}^{2}\right)+\cdots, \\
& S_{3 \text {-loop }}^{C} / N^{2}=-\beta \frac{\lambda^{2}}{16 M^{5}} D(D-1)^{2}-\frac{\beta \lambda^{2}}{8 M^{5}} D(D-1)^{2}\left(\beta M x(x+1)^{2}+x^{3}+2 x^{2}+3 x\right)\left|u_{1}\right|^{2} \\
& -\frac{\beta \lambda^{2}}{8 M^{5}} D(D-1)^{2}\left(2 \beta M x^{4}+x^{4}+2 x^{2}\right)\left|u_{1}\right|^{4}-\frac{\beta \lambda^{2}}{8 M^{5}} D(D-1)^{2}\left(2 \beta M\left(x^{6}+2 x^{4}+x^{2}\right)+x^{6}+2 x^{4}+3 x^{2}\right)\left|u_{2}\right|^{2} \\
& -\frac{\beta \lambda^{2}}{8 M^{5}} D(D-1)^{2}\left(\beta M\left(2 x^{5}+x^{4}+4 x^{3}+x^{2}\right)+x^{5}+x^{4}+3 x^{3}+x^{2}\right)\left(u_{2} u_{-1}^{2}+u_{-2} u_{1}^{2}\right)+\cdots .
\end{aligned}
$$

In addition, we need to compute

$$
\begin{aligned}
- & \left\langle\int_{0}^{\beta} d t \operatorname{Tr}\left(-\frac{M^{2}}{2}\left(X^{I}\right)^{2}\right) \int_{0}^{\beta} d t^{\prime} \operatorname{Tr}\left(-\frac{g^{2}}{4}\left[X^{I}, X^{J}\right]^{2}\right)\right\rangle_{\text {connected }}=-M^{2} \frac{\partial}{\partial M^{2}}\left\langle\int_{0}^{\beta} d t \operatorname{Tr}\left(-\frac{g^{2}}{4}\left[X^{I}, X^{J}\right]^{2}\right)\right\rangle \\
= & \frac{\beta N^{2} \lambda}{8 M^{2}} D(D-1)+\frac{\beta N^{2} \lambda}{4 M^{2}} D(D-1) \sum_{n=1}^{\infty}\left((n \beta M+1) x^{2 n}+(2+n \beta M) x^{n}\right)\left|u_{n}\right|^{2} \\
& +\frac{\beta N^{2} \lambda}{8 M^{2}} D(D-1)\left((\beta M+1) x^{2}+(3 \beta M+2) x^{3}\right)\left(u_{1}^{2} u_{-2}+u_{-1}^{2} u_{2}\right)+\cdots
\end{aligned}
$$

and

$$
\begin{aligned}
- & \frac{1}{2}\left\langle\int_{0}^{\beta} d t \operatorname{Tr}\left(-\frac{M^{2}}{2}\left(X^{I}\right)^{2}\right) \int_{0}^{\beta} d t^{\prime} \operatorname{Tr}\left(-\frac{M^{2}}{2}\left(X^{I}\right)^{2}\right)\right\rangle_{\text {connected }} \\
& =\frac{1}{2} M^{4} \frac{\partial^{2}}{\partial\left(M^{2}\right)^{2}} S_{1-\text { loop }}=-\beta \frac{D N^{2} M}{16}-\frac{D N^{2}}{8} \sum_{n=1}^{\infty}\left(n(\beta M)^{2}+\beta M\right) x^{n}\left|u_{n}\right|^{2} .
\end{aligned}
$$

The three-loop correction $S_{3 \text {-loop }}$ in (A1) is given as the sum of (A7), (A11) and (A12).

By substituting these results to (A1), we can read off the effective action at three-loop order,

$$
S_{\text {eff }}\left(\left\{u_{n}\right\}, M\right)=N^{2}\left(\beta f_{0}+f_{1}\left|u_{1}\right|^{2}+f_{2}\left|u_{1}\right|^{4}+f_{3}\left|u_{2}\right|^{2}+f_{4}\left(u_{2} u_{-1}^{2}+u_{-2} u_{1}^{2}\right)+\cdots\right)
$$

Here

$$
f_{0}=\frac{D M}{2}+\kappa\left(\frac{\lambda}{8 M^{2}} D(D-1)-\frac{D M}{4}\right)+\kappa^{2}\left[-\frac{3 \lambda^{2}}{128 M^{5}} D(D-1)-\frac{3 \lambda^{2}}{32 M^{5}} D(D-1)^{2}+\frac{\lambda}{8 M^{2}} D(D-1)-\frac{D M}{16}\right]
$$




$$
\begin{aligned}
f_{1}= & 1-D x+\kappa\left(\frac{\beta \lambda}{4 M^{2}} D(D-1)\left(x^{2}+2 x\right)-\frac{1}{2} \beta D M x\right)+\kappa^{2}\left[-\frac{3 \beta \lambda^{2}}{32 M^{5}} D(D-1)\left(2 \beta M x^{2}-x^{3}+x^{2}+3 x\right)\right. \\
& -\frac{\beta \lambda^{2}}{16 M^{5}} D(D-1)^{2}\left(\beta M\left(2 x^{2}+x\right)+3 x^{2}+3 x\right)-\frac{\beta \lambda^{2}}{8 M^{5}} D(D-1)^{2}\left(\beta M x(x+1)^{2}+x^{3}+2 x^{2}+3 x\right) \\
& \left.+\frac{\beta \lambda}{4 M^{2}} D(D-1)\left(x^{2}(\beta M+1)+x(\beta M+2)\right)-\frac{1}{8} D x\left((\beta M)^{2}+\beta M\right)\right], \\
f_{2}= & \kappa^{2}\left[-\frac{3 \beta \lambda^{2}}{64 M^{5}} D(D-1)\left(2 \beta M x^{2}-2 x^{4}+5 x^{2}\right)-\frac{3 \beta \lambda^{2}}{16 M^{5}} D(D-1)^{2}\left(\beta M x^{3}+x^{3}\right)-\frac{\beta \lambda^{2}}{8 M^{5}} D(D-1)^{2}\left(2 \beta M x^{4}+x^{4}+2 x^{2}\right)\right], \\
f_{3}= & \frac{1}{2}\left(1-D x^{2}\right)+\kappa\left(\frac{\beta \lambda}{4 M^{2}} D(D-1)\left(x^{4}+2 x^{2}\right)-\frac{1}{2} \beta D M x^{2}\right)+\kappa^{2}\left[-\frac{D}{8}\left(2(\beta M)^{2}+\beta M\right) x^{2}\right. \\
+ & \frac{\beta \lambda}{4 M^{2}} D(D-1)\left((2 \beta M+1) x^{4}+(2+2 \beta M) x^{2}\right)-\frac{\beta \lambda^{2}}{8 M^{5}} D(D-1)^{2}\left(2 \beta M\left(x^{6}+2 x^{4}+x^{2}\right)+x^{6}+2 x^{4}+3 x^{2}\right) \\
& \left.-\frac{\beta \lambda^{2}}{16 M^{5}} D(D-1)^{2}\left(2 \beta M\left(2 x^{4}+x^{2}\right)+3 x^{4}+3 x^{2}\right)-\frac{3 \beta \lambda^{2}}{32 M^{5}} D(D-1)\left(4 \beta M x^{4}-x^{6}+x^{4}+3 x^{2}\right)\right], \\
f_{4}= & \kappa \frac{\beta \lambda}{8 M^{2}} D(D-1)\left(x^{2}+2 x^{3}\right)+\kappa^{2}\left[\frac{\beta \lambda}{8 M^{2}} D(D-1)\left((\beta M+1) x^{2}+(3 \beta M+2) x^{3}\right)\right. \\
& -\frac{3 \beta \lambda^{2}}{32 M^{5}} D(D-1)\left(4 \beta M x^{3}-x^{5}-x^{4}+3 x^{3}+2 x^{2}\right)-\frac{\beta \lambda^{2}}{32 M^{5}} D(D-1)^{2}\left(2 \beta M\left(2 x^{4}+3 x^{3}+x^{2}\right)+3 x^{4}+6 x^{3}+3 x^{2}\right) \\
& \left.-\frac{\beta \lambda^{2}}{8 M^{5}} D(D-1)^{2}\left(\beta M\left(2 x^{5}+x^{4}+4 x^{3}+x^{2}\right)+x^{5}+x^{4}+3 x^{3}+x^{2}\right)\right] .
\end{aligned}
$$

We have used $x=e^{-\beta M}$. If we are interested in the twoloop effective action, we should simply ignore $O\left(\kappa^{2}\right)$ terms in this result.

\section{APPENDIX B: THE DETAILS OF THE DERIVATION OF THE CRITICAL DIMENSION}

To show the details of the derivations of the critical dimension in Sec. IIC, we analyze the effective action (A13) and discuss how we determine the phase structure. We will mainly show the analysis at two-loop, since the three-loop analysis is almost parallel. [Recall that we remove $O\left(\kappa^{2}\right)$ terms in (A13) when we consider the two-loop effective theory.] We set $\kappa=1$ in (A13) hereafter to use the principle of the minimum sensitivity.

First, we consider a low temperature regime. There, $x=e^{-\beta M}$ would be small, and $f_{1}$ and $f_{3}$ would be positive. Then, to make the effective action (A13) small, $\left\langle u_{1}\right\rangle=$ $\left\langle u_{2}\right\rangle=0$ would be favored. Thus, the effective action (A13) would become $S_{\text {eff }}=\beta N^{2} f_{0}(M)$.

Here, we need to determine $M$. As we have discussed in (5), we fix $M$ such that the $M$ dependence of the effective action is minimized. From (A14), we find that at

$$
M_{0}=\lambda^{1 / 3}(D-1)^{1 / 3},
$$

$\partial_{M} f_{0}$ at two-loop becomes 0 and is minimized. Then we obtain the free energy at low temperatures as

$$
F=S_{\text {eff }} / \beta=N^{2} f_{0}\left(M_{0}\right)=\frac{3}{8} N^{2} D \lambda^{1 / 3}(D-1)^{1 / 3} .
$$

This result is shown in Fig. 2 and Table II. We find good agreement with the MC results even at two-loop order.

Next, in order to investigate the phase transition, we compute $M_{i}$ and $\bar{f}_{i}$ defined in (9) and (11). However, since $\partial_{M} f_{0}=0$ at $M=M_{0}$, we obtain $\bar{f}_{i}=f_{i}$ for $i=1,3,4$ and we need to evaluate only $M_{1}$ and $\bar{f}_{2}$. By substituting the expansion (9) into the equation $\partial_{M} S_{\text {eff }}=0$, we find

$$
M_{1}=-\frac{\partial_{M} f_{1}}{\beta \partial_{M}^{2} f_{0}}, \quad \bar{f}_{2}=-\frac{1}{2} \frac{\left(\partial_{M} f_{1}\right)^{2}}{\beta \partial_{M}^{2} f_{0}} .
$$

The explicit formulas for these equations are rather messy, and we omit showing them, but one can obtain them easily by using Mathematica.

Now, we are ready to discuss the critical phenomena. As we have argued below (12), the critical temperature can be found through 


$$
0=a(T)=1-D e^{-\beta M_{0}}+\frac{D}{4} \beta M_{0} e^{-2 \beta M_{0}}
$$

This equation can be solved numerically and the result is summarized in Fig. 2 and Table III. Again our results seem to be consistent with the MC results.

Finally, we determine the order of the phase transition. Through the discussions around (13), it is determined by the signature of $b$ defined in (12) at the critical temperature. We numerically see that it is always negative as shown in Fig. 4 and indicates the first order phase transition for any $D$ at two-loop order. [As we will shown in (D9), we can confirm it analytically, if $D$ is large.]

So far, we have shown the two-loop results. Now we will move on to the three-loop case. The three-loop calculation is almost parallel to the two-loop analysis. One significant difference is that the minimum of $\left|\partial_{M} f_{0}\right|$ in (5) is not zero. Hence we need to find the minimum via $\partial_{M}^{2} f_{0}=0$, and obtain

$$
M_{0}=\frac{15^{1 / 3} \lambda^{1 / 3}}{2}(D-3 / 4)^{1 / 3}
$$

The rest of the calculations are straightforward. We obtain the free energy in the confinement phase as

$$
F=N^{2} f_{0}\left(M_{0}\right)=N^{2} \lambda^{1 / 3} \frac{D(1412 D-1187)}{160(30(4 D-3))^{2 / 3}} .
$$

This result is shown in Fig. 2 and Table II.

Next, we fix $M_{i}$ via $\partial_{M}^{2} S_{\text {eff }}=0$ near the critical temperature, and obtain

$$
\begin{aligned}
M_{i}= & -\frac{\partial_{M}^{2} f_{i}}{\beta \partial_{M}^{3} f_{0}}, \quad(i=1,3,4), \\
M_{2}= & -\frac{1}{\beta \partial_{M}^{3} f_{0}}\left(\partial_{M}^{2} f_{2}-\partial_{M}^{3} f_{1} \frac{\partial_{M}^{2} f_{1}}{\beta \partial_{M}^{3} f_{0}}\right. \\
& \left.+\frac{\partial_{M}^{4} f_{0}}{2 \beta}\left(\frac{\partial_{M}^{2} f_{1}}{\partial_{M}^{3} f_{0}}\right)^{2}\right),
\end{aligned}
$$

where $f_{i}$ are evaluated at $M=M_{0}$. Then $\bar{f}_{i}, a$ and $b$ are derived through (11) and (12). Finally, by solving $a\left(T_{c}\right)=0$ and evaluating $b\left(T_{c}\right)$ numerically, we obtain the critical temperatures and the orders of the phase transitions as shown in Fig. 2 and 4.

\section{APPENDIX C: OTHER OBSERVABLES AND SPECIFIC HEAT}

We can also compute other observables via our analysis. For example, the vevs of the square of the adjoint scalars $X^{I}$, which have been investigated in the MC studies [28,31], can be derived as
TABLE V. $R^{2}$ defined in (C1) in the confinement phase. We have used the unit $\lambda=1$. The two-loop and three-loop results are from $(\mathrm{C} 2)$ and $(\mathrm{C} 3)$, respectively. The $1 / D$ expansion results are from (D2). The MC results are from the unpublished data in [31].

\begin{tabular}{lcccc}
\hline \hline$D$ & Two-loop & Three-loop & $1 / D$ expansion & $\mathrm{MC}(T=0.50)$ \\
\hline 2 & 1.0 & 0.969 & 0.996 & $1.15(N=60)$ \\
3 & 1.19 & 1.17 & 1.41 & $1.31(N=32)$ \\
4 & 1.39 & 1.37 & 1.42 & $1.45(N=32)$ \\
5 & 1.57 & 1.56 & 1.61 & $1.62(N=24)$ \\
6 & 1.75 & 1.74 & 1.79 & $1.81(N=32)$ \\
9 & 2.25 & 2.24 & 2.28 & $2.29(N=32)$ \\
13 & 2.84 & 2.83 & 2.87 & $2.87(N=32)$ \\
\hline \hline
\end{tabular}

$$
\begin{aligned}
R^{2} & :=\frac{g^{2}}{N}\left\langle\operatorname{Tr} X^{I} X^{I}\right\rangle \\
& =\left.\frac{2 \lambda}{\beta M^{2}}\left(-\kappa \partial_{\kappa} S_{\text {eff }}+\lambda \partial_{\lambda} S_{\text {eff }}\right)\right|_{\kappa \rightarrow 1},
\end{aligned}
$$

where $S_{\text {eff }}$ is the effective action (A1). In the confinement phase, it can be calculated as

$$
\begin{gathered}
R^{2}=\frac{\lambda^{2 / 3} D}{2(D-1)^{1 / 3}}, \quad \text { (two-loop) } \\
R^{2}=\frac{\lambda^{2 / 3} D(148 D-103)}{15 \times 30^{1 / 3}(4 D-3)^{4 / 3}}, \quad \text { (three-loop). }
\end{gathered}
$$

These quantities agree with the MC studies [31] as shown in Table V.

We can also compute specific heat $C$ from the free energy through the ordinary thermodynamical relation, and we obtain

$$
\begin{aligned}
C / N^{2} & =\partial_{T} E=O\left(1 / N^{2}\right), \quad\left(T<T_{c}, u_{1}=0\right), \\
& =\frac{T_{c}^{2} c^{2}}{2 b} \quad\left(T=T_{c}, u_{1} \neq 0\right),
\end{aligned}
$$

where we have used (14) and

$$
b=\bar{f}_{2}-\frac{\bar{f}_{4}^{2}}{\bar{f}_{3}}, \quad c=\frac{\partial a(T)}{\partial T} .
$$

[ $a, b$, and $\bar{f}_{i}$ are defined in (11) and (12).] The specific heat is very small in the confinement phase due to the large- $N$ volume independence, which strongly suppresses temperature dependence of physical quantities [16,52]. It becomes positive in the second order phase transition case, since $b>0$, while it becomes negative in the first order transition case $(b<0)$. Phases with negative specific heat are unphysical in usual thermodynamical systems. However, in our case, it becomes physical in the microcanonical ensemble [24]. (See [41-43] for some discussions on phases with negative specific heat.) Also, the specific 
heat (C4) at the critical temperature tends to diverge as $D$ approaches to the critical dimension $D=35.5$, where $b$ crosses 0 . (Of course, $D$ is digit, and $b$ cannot be 0 .) The result is summarized in Fig. 5 and Table IV.

\section{APPENDIX D: LARGE-D LIMIT}

At large- $D$, the $1 / D$ expansion [30] would be reliable. Hence, it would be valuable to evaluate our results at large$D$ and compare them with the $1 / D$ expansion [30].

In the large- $D$ expansion, we obtain the following quantities:

$$
\begin{aligned}
& F /\left.N^{2}\right|_{\beta \rightarrow \infty}= D(\lambda D)^{1 / 3} \\
& \times\left(\frac{3}{8}+\frac{1}{D}\left(-\frac{81}{64}+\frac{\sqrt{5}}{2}\right)+O\left(1 / D^{2}\right)\right), \\
&\left.R^{2}\right|_{\beta \rightarrow \infty}=(\lambda D)^{2 / 3}\left(\frac{1}{2}+\frac{1}{D}\left(\frac{7 \sqrt{5}}{30}-\frac{9}{32}\right)+O\left(1 / D^{2}\right)\right), \\
& \beta_{c}=\frac{\log D}{(\lambda D)^{1 / 3}}\left(1+\frac{1}{D}\left(\frac{203}{160}-\frac{\sqrt{5}}{3}\right)+O\left(1 / D^{2}\right)\right) .
\end{aligned}
$$

These are from (4.27), (4.33) with (4.25) and (4.30) in [30], respectively. Besides, we evaluate $b\left(T_{c}\right)$ in the effective action (12), which fixes the order of the transition, as

$$
\begin{aligned}
\left.b\right|_{T=T_{c}}= & \frac{\log D}{D}\left(\frac{1}{3}+\frac{1}{D}\left(\frac{1049}{600}-\frac{197 \sqrt{5}}{600}-\frac{33}{400} \log D\right)\right. \\
& \left.+O\left(1 / D^{2}\right)\right)
\end{aligned}
$$

where we have used (4.29) and (4.30) in [30]. This is always positive and the $1 / D$ expansion predicts the second order phase transition at large- $D$. Through $(\mathrm{C} 4)$, we obtain the specific heat at $T=T_{c}$ as

$$
\begin{aligned}
C /\left.N^{2}\right|_{T=T_{c}}= & \frac{3}{2} D \log D-\frac{3 \log D}{800}(2098-394 \sqrt{5} \\
& -99 \log D)+O(1 / D) .
\end{aligned}
$$

We will compare these quantities with our results at large- $D$.
First, we evaluate our two-loop results at large- $D$. At two-loop, we can solve (B4) at large- $D$ and obtain the critical temperature analytically. Then, we obtain

$$
\begin{gathered}
F /\left.N^{2}\right|_{\beta \rightarrow \infty}=D(\lambda D)^{1 / 3}\left(\frac{3}{8}-\frac{1}{8 D}+O\left(1 / D^{2}\right)\right), \\
\left.R^{2}\right|_{\beta \rightarrow \infty}=(\lambda D)^{2 / 3}\left(\frac{1}{2}+\frac{1}{6 D}+O\left(1 / D^{2}\right)\right), \\
\beta_{c}=\frac{\log D}{(\lambda D)^{1 / 3}}\left(1+\frac{1}{12 D}+O\left(1 / D^{2}\right)\right), \\
\left.b\right|_{T=T_{c}}=-\frac{1}{6 D} \log D(1+O(1 / D)), \\
C /\left.N^{2}\right|_{T=T_{c}}=-3 D \log D(1+O(1 / D)) .
\end{gathered}
$$

Thus, $b$ is negative, and it does not agree with the $1 / D$ expansion (D4). On the other hand, the leading order terms of $F, R^{2}$ and $\beta_{c}$ in our results are precisely coincident with those of the $1 / D$ expansion, although the $1 / D$ corrections differ. Since the results of the $1 / D$ expansion [30] would be reliable at large- $D$, these quantities at two-loop order are accidentally very good at large- $D$.

Next, we consider the three-loop results. Different from the two-loop case, we cannot solve $T_{c}$ in the three-loop case analytically even at large- $D$. From (B6) and (C3), we obtain

$$
\begin{gathered}
F /\left.N^{2}\right|_{\beta \rightarrow \infty}=D(\lambda D)^{1 / 3}\left(\frac{1412}{160(120)^{2 / 3}}+O(1 / D)\right), \\
\left.R^{2}\right|_{\beta \rightarrow \infty}=(\lambda D)^{2 / 3}\left(\frac{148}{60(120)^{1 / 3}}+O(1 / D)\right) .
\end{gathered}
$$

Thus, they do not agree with (D1) and (D2) in the $1 / D$ expansion. However, these are numerically not bad. For $F$, if we compare the coefficients of the leading terms of (D1) and (D11), we obtain $3 / 8=0.375$ and $1412 / 160(120)^{2 / 3}=0.363 \ldots$ and the error is $3 \%$ only. Similarly, for $R^{2}$, we have $1 / 2=0.5$ and $148 / 60(120)^{1 / 3}=0.500092 \ldots$, and they are very close. Hence, we presume that the convergence of the principle of the minimum sensitivity at large- $D$ would be good in our model. 
[1] R. Gregory and R. Laflamme, The instability of charged black strings and p-branes, Nucl. Phys. B428, 399 (1994).

[2] B. Kol, The phase transition between caged black holes and black strings: A review, Phys. Rep. 422, 119 (2006).

[3] E. Sorkin, A Critical Dimension in the Black String Phase Transition, Phys. Rev. Lett. 93, 031601 (2004).

[4] H. Kudoh and U. Miyamoto, On nonuniform smeared black branes, Classical Quantum Gravity 22, 3853 (2005).

[5] V. Cardoso and O. J. C. Dias, Rayleigh-Plateau and Gregory-Laflamme Instabilities of Black Strings, Phys. Rev. Lett. 96, 181601 (2006).

[6] U. Miyamoto and K.-i. Maeda, Liquid bridges and black strings in higher dimensions, Phys. Lett. B 664, 103 (2008).

[7] J. M. Maldacena, The Large $N$ limit of superconformal field theories and supergravity, Int. J. Theor. Phys. 38, 1113 (1999); Adv. Theor. Math. Phys. 2, 231 (1998).

[8] N. Itzhaki, J. M. Maldacena, J. Sonnenschein, and S. Yankielowicz, Supergravity and the large N limit of theories with sixteen supercharges, Phys. Rev. D 58, 046004 (1998).

[9] O. Aharony, J. Marsano, S. Minwalla, and T. Wiseman, Black hole-black string phase transitions in thermal $1+1$ dimensional supersymmetric Yang-Mills theory on a circle, Classical Quantum Gravity 21, 5169 (2004).

[10] S. Catterall, A. Joseph, and T. Wiseman, Thermal phases of D1-branes on a circle from lattice super Yang-Mills, J. High Energy Phys. 12 (2010) 022.

[11] G. Mandal and T. Morita, Phases of a two dimensional large N gauge theory on a torus, Phys. Rev. D 84, 085007 (2011).

[12] G. Mandal and T. Morita, Gregory-Laflamme as the confinement/deconfinement transition in holographic QCD, J. High Energy Phys. 09 (2011) 073.

[13] T. Morita, S. Shiba, T. Wiseman, and B. Withers, Moduli dynamics as a predictive tool for thermal maximally supersymmetric Yang-Mills at large N, J. High Energy Phys. 07 047 (2015).

[14] A. J. C. Dias, J. E. Santos, and B. Way, Localised and nonuniform thermal states of super-Yang-Mills on a circle, J. High Energy Phys. 06 (2017) 029.

[15] S. Catterall, R. G. Jha, D. Schaich, and T. Wiseman, Testing holography using lattice super-Yang-Mills theory on a 2-torus, Phys. Rev. D 97, 086020 (2018).

[16] T. Eguchi and H. Kawai, Reduction of Dynamical Degrees of Freedom in the Large $N$ Gauge Theory, Phys. Rev. Lett. 48, 1063 (1982).

[17] B. de Wit, J. Hoppe, and H. Nicolai, On the quantum mechanics of supermembranes, Nucl. Phys. B305, 545 (1988).

[18] T. Banks, W. Fischler, S. H. Shenker, and L. Susskind, $M$ theory as a matrix model: A conjecture, Phys. Rev. D 55, 5112 (1997).

[19] D. E. Berenstein, J. M. Maldacena, and H. S. Nastase, Strings in flat space and pp waves from $N=4$ super Yang-Mills, J. High Energy Phys. 04 (2002) 013.

[20] O. Aharony, J. Marsano, S. Minwalla, K. Papadodimas, M. Van Raamsdonk, and T. Wiseman, The phase structure of low dimensional large $\mathrm{N}$ gauge theories on Tori, J. High Energy Phys. 01 (2006) 140.

[21] K. Hashimoto, N. Iizuka, and P. Yi, A matrix model for baryons and nuclear forces, J. High Energy Phys. 10 (2010) 003.
[22] K. Hashimoto and T. Morita, Nucleus from string theory, Phys. Rev. D 84, 046004 (2011).

[23] B. Sundborg, The Hagedorn transition, deconfinement and $N=4$ SYM theory, Nucl. Phys. B573, 349 (2000).

[24] O. Aharony, J. Marsano, S. Minwalla, K. Papadodimas, and M. Van Raamsdonk, The Hagedorn-deconfinement phase transition in weakly coupled large $\mathrm{N}$ gauge theories, Adv. Theor. Math. Phys. 8, 603 (2004).

[25] D. N. Kabat and G. Lifschytz, Approximations for strongly coupled supersymmetric quantum mechanics, Nucl. Phys. B571, 419 (2000).

[26] L. Alvarez-Gaume, C. Gomez, H. Liu, and S. R. Wadia, Finite temperature effective action, ads $_{5}$ black holes, and 1/ $n$ expansion, Phys. Rev. D 71, 124023 (2005).

[27] M. Hanada and T. Nishioka, Cascade of Gregory-Laflamme transitions and U(1) breakdown in super Yang-Mills, J. High Energy Phys. 09 (2007) 012.

[28] N. Kawahara, J. Nishimura, and S. Takeuchi, Phase structure of matrix quantum mechanics at finite temperature, J. High Energy Phys. 10 (2007) 097.

[29] T. Azeyanagi, M. Hanada, T. Hirata, and H. Shimada, On the shape of a $D$-brane bound state and its topology change, J. High Energy Phys. 03 (2009) 121.

[30] G. Mandal, M. Mahato, and T. Morita, Phases of one dimensional large $\mathrm{N}$ gauge theory in a 1/D expansion, J. High Energy Phys. 02 (2010) 034.

[31] T. Azuma, T. Morita, and S. Takeuchi, Hagedorn Instability in Dimensionally Reduced Large-N Gauge Theories as Gregory-Laflamme and Rayleigh-Plateau Instabilities, Phys. Rev. Lett. 113, 091603 (2014).

[32] V. G. Filev and D. O'Connor, The BFSS model on the lattice, J. High Energy Phys. 05 (2016) 167.

[33] M. Hanada and P. Romatschke, Lattice Simulations of 10d Yang-Mills toroidally compactified to 1d, 2d and 4d, Phys. Rev. D 96, 094502 (2017).

[34] G. Bergner, N. Bodendorfer, M. Hanada, E. Rinaldi, and A. Schäfer, and P. Vranas, Thermal phase transition in YangMills matrix model, J. High Energy Phys. 01 (2020) 053.

[35] Y. Asano, S. Kováčic, and D. O'Connor, The confining transition in the bosonic bmn matrix model, arXiv: 2001.03749.

[36] Generally, it is nontrivial to compute such a D-brane distribution in matrix models [22,29,37].

[37] K. Hashimoto, The shape of non-Abelian D branes, J. High Energy Phys. 04 (2004) 004.

[38] More precisely, the temporal direction in the gravity is also periodic in order to make the system to be at a finite temperature. This temporal direction corresponds to the T-dual of one of $X^{I}$, say $X^{1}$, in the matrix model. If the temperature in the gravity is sufficiently high, the size of the $S^{1}$ after the T-dual is large, and we can ignore the periodicity of $X^{1}$ in the matrix model [9].

[39] P. M. Stevenson, Optimized perturbation theory, Phys. Rev. D 23, 2916 (1981).

[40] It has been discussed that, generally, some long-range interactions make phases having negative specific heat possible [41-43]. In our case, presumably, the interactions between the diagonal components of the matrices may be regarded as long range, since no screening effect occurs, and the negative specific heat realizes. 
[41] W. Thirring, Systems with negative specific heat, Z. Phys. A 235, 339 (1970).

[42] Y. Levin, R. Pakter, F. B. Rizzato, T. N. Teles, and F. P. Benetti, Nonequilibrium statistical mechanics of systems with long-range interactions, Phys. Rep. 535, 1 (2014).

[43] D. Berenstein, Negative specific heat from nonplanar interactions and small black holes in AdS/CFT, J. High Energy Phys. 10 (2019) 001.

[44] S. W. Hawking and D. N. Page, Thermodynamics of black holes in anti-de Sitter space, Commun. Math. Phys. 87, 577 (1983).

[45] There are several studies, which apply the principle of minimum sensitivity to YM type matrix models [46-50].

[46] K. Hashimoto, Y. Matsuo, and T. Morita, Nuclear states and spectra in holographic QCD, J. High Energy Phys. 12 (2019) 001.

[47] J. Nishimura and F. Sugino, Dynamical generation of fourdimensional space-time in the IIB matrix model, J. High Energy Phys. 05 (2002) 001.

[48] H. Kawai, S. Kawamoto, T. Kuroki, T. Matsuo, and S. Shinohara, Mean field approximation of IIB matrix model and emergence of four-dimensional space-time, Nucl. Phys. B647, 153 (2002).

[49] T. Aoyama, J. Nishimura, and T. Okubo, Spontaneous breaking of the rotational symmetry in dimensionally reduced super Yang-Mills models, Prog. Theor. Phys. 125, 537 (2011).

[50] J. Nishimura, T. Okubo, and F. Sugino, Systematic study of the $\mathrm{SO}(10)$ symmetry breaking vacua in the matrix model for type IIB superstrings, J. High Energy Phys. 10 (2011) 135.

[51] $f_{0}$ does not depend on temperature. This is a consequence of the large- $N$ volume independence $[16,52]$. In the confinement phase, all $u_{n}=0$, and the free energy is given by
$N^{2} f_{0}$. The large- $N$ volume independence ensures that the free energy is independent of temperature.

[52] A. Gocksch and F. Neri, On Large $N$ QCD at Finite Temperature, Phys. Rev. Lett. 50, 1099 (1983).

[53] We need at least two-loop to apply the principle of minimum sensitivity.

[54] Because of the large- $N$ volume independence [16,52], the temperature dependences of the observables in the confinement phase are very small. Hence we omit to show temperatures of the MC results in the confinement phase in this article.

[55] In the $1 / D$ expansion [30], it has been shown that the scalar fields $X^{I}$ acquire a mass dynamically. We guess that the reason for the quantitative success in reproducing the MC results through our analysis is that the mass deformation (3) may appropriately capture this dynamical mass.

[56] D. J. Gross and E. Witten, Possible third order phase transition in the large $N$ lattice gauge theory, Phys. Rev. D 21, 446 (1980).

[57] S. R. Wadia, A study of U(N) lattice gauge theory in two dimensions, arXiv:1212.2906.

[58] G. Mandal and T. Morita, Quantum quench in matrix models: Dynamical phase transitions, selective equilibration and the generalized Gibbs ensemble, J. High Energy Phys. 10 (2013) 197.

[59] In the second order phase transition case $(D \geq 36)$, the stable configuration just above $T_{c}$ is the nonuniform distribution [20,24,26,30].

[60] H. Kudoh and T. Wiseman, Connecting Black Holes and Black Strings, Phys. Rev. Lett. 94, 161102 (2005).

[61] P. Figueras, K. Murata, and H. S. Reall, Stable nonuniform black strings below the critical dimension, J. High Energy Phys. 11 (2012) 071. 